\title{
Stability of KcsA Tetramer Depends on Membrane Lateral Pressure ${ }^{\dagger}$
}

\author{
Els van den Brink-van der Laan,* Vladimir Chupin, J. Antoinette Killian, and Ben de Kruijff \\ Department Biochemistry of Membranes, Centre for Biomembranes and Lipid Enzymology, Institute of Biomembranes, \\ Utrecht University, Padualaan 8, 3584 CH, Utrecht, The Netherlands
}

Received November 26, 2003; Revised Manuscript Received February 12, 2004

\begin{abstract}
The potassium channel KcsA forms an extremely stable tetramer. Despite this high stability, it has been shown that the membrane-mimicking solvent 2,2,2-trifluoroethanol (TFE) can induce tetramer dissociation [Valiyaveetil, F. I., et al. (2002) Biochemistry 41, 10771-7, and Demmers, J. A. A., et al. (2003) FEBS Lett. 541, 69-77]. Here we have studied the effect of TFE on the structure and oligomeric state of the KcsA tetramer, reconstituted in different lipid systems. It was found that TFE changes the secondary and tertiary structure of KcsA and that it can dissociate the KcsA tetramer in all systems used. The tetramer is stabilized by a lipid bilayer as compared to detergent micelles. The extent of stabilization was found to depend on the nature of the lipids: a strong stabilizing effect of the nonbilayer lipid phosphatidylethanolamine (PE) was observed, but no effect of the charged phoshosphatidylglycerol (PG) as compared to phosphatidylcholine (PC) was found. To understand how lipids stabilize KcsA against TFE-induced tetramer dissociation, we also studied the effect of TFE on the bilayer organization in the various lipid systems, using ${ }^{31} \mathrm{P}$ and ${ }^{2} \mathrm{H}$ NMR. The observed lipid dependency was similar as was found for tetramer stabilization: PE increased the bilayer stability as compared to PC, while PG behaved similar to PC. Furthermore, it was found that TFE has a large effect on the acyl chain ordering. The results indicate that TFE inserts primarily in the membrane interface. We suggest that the lipid bilayer stabilizes the KcsA tetramer by the lateral pressure in the acyl chain region and that this stabilizing effect increases when a nonbilayer lipid like PE is present.
\end{abstract}

Many membrane proteins are active as stable oligomers. The assembly of these oligomeric proteins is poorly understood. The protein subunits interact via protein-protein interactions, for which some oligomerization motifs have been identified $(1-3)$. Besides protein-protein interactions, protein-lipid interactions can play an important role in oligomerization. Membrane protein complexes can interact with a specific type of lipid at a particular position, as was suggested for instance by the presence of lipids in the crystal structure of a number of membrane proteins (4-7). An example is the light harvesting complex of photosystem II, which contains phosphatidylglycerol $(\mathrm{PG})^{1}$ (4). For this protein, it was shown that delipidation results in dissociation of the trimeric complex, suggesting an important role of PG

\footnotetext{
$\dagger$ This work was supported by the Dutch Foundation for Fundamental Research on Matter (FOM).

* Corresponding author. Fax: (31)(30)2533969. Tel.: (31)(30)2532465. E-mail: e.vandenbrink@chem.uu.nl.

${ }^{1}$ Abbreviations: CD, circular dichroism; CF, carboxyfluorescein; CSA, chemical shift anisotropy; DDM, $n$-dodecyl- $\beta$-D-maltoside; DEPE, 1,2-dielaidoyl- $s n$-glycero-3-phosphoethanolamine; DOPC, 1,2-dioleoyl$s n$-glycero-3-phosphocholine; DOPE, 1,2-dioleoyl-sn-glycero-3-phosphoethanolamine; DOPG, 1,2-dioleoyl-sn-glycero-3-phosphoglycerol; $\Delta v_{\mathrm{q}}$, quadrupolar splitting; E. coli, Escherichia coli; ${ }^{2} \mathrm{H}_{4}$-DOPC, DOPC, labeled with two deuterium atoms on both acyl chains at the $\mathrm{C}_{11}$ position; ESI-MS, electrospray ionization mass spectrometry; $\mathrm{H}_{\mathrm{II}}$, inverted hexagonal phase; $\mathrm{L}_{\alpha}$, liquid crystalline lamellar phase; LUVs, large unilamellar vesicles prepared by extrusion techniques; mdeg, millidegrees; NMR, nuclear magnetic resonance; NRMSD, normalized root-mean-square deviation; PC, phosphatidylcholine; PE, phosphatidylethanolamine; PG, phosphatidylglycerol; SDS-PAGE, sodium dodecylsulphate-polyacrylamide gel electrophoresis; TFE, 2,2,2trifluoroethanol; $\theta$, mean residue ellipticity.
}

in the stabilization of the oligomeric protein complex (4). Also nonspecific interactions can be important for the stability and function of membrane protein complexes, depending on more general properties of lipids, such as the charge of the lipid headgroup (8), the extent of hydrophobic mismatch (9), or the presence of nonbilayer lipids $(10,11)$.

The potassium channel KcsA is an oligomeric membrane protein from Streptomyces lividans, which is a popular model protein to study ion channel function $(12-15)$ and more recently membrane protein assembly $(16-21)$. For this tetrameric protein, both specific and general protein-lipid interactions seem to be important. Each subunit contains two transmembrane segments, connected by the pore region, a large C-terminal cytoplasmic domain and an $\mathrm{N}$-terminal domain at the membrane interface $(22,23)$. It has been shown that the nonbilayer lipid phosphatidylethanolamine (PE) and the charged lipid PG are important for efficient membrane association and tetramerization of KcsA, while the assembly of KcsA was much less supported by the zwitterionic bilayer lipid phosphatidylcholine (PC) (19). PG could be copurified with KcsA and was suggested to be present in the crystal structure of KcsA (24). Also by mass spectrometry a protein-lipid complex of KcsA with PG and, to a lesser extent, with PE was observed (25). These interactions may be either specific or they may be more general, for example, based on electrostatic interactions in the case of PG or on the propensity to form nonbilayer structures in the case of PE.

The KcsA tetramer is extremely stable, even in a detergent such as SDS (26). However, an unexpected observation was 
made that in the presence of 2,2,2-trifluoroethanol (TFE) only monomeric KcsA can be measured by electrospray ionization mass spectrometry (ESI-MS) and SDS-PAGE (24, 25), suggesting that TFE induces dissociation of the KcsA tetramer. These observations were surprising since TFE is often used as a membrane mimicking solvent and is known for its ability to stabilize protein secondary structure (especially $\alpha$-helices) (27). Although the KcsA tetramer seemed to dissociate in the presence of TFE, specific protein-lipid interactions could still be measured by ESI-MS, preferentially with PG and PE (25).

We hypothesize that besides protein-protein interactions protein-lipid interactions also are involved in the stabilization of the KcsA tetramer, and that especially these latter interactions are weakened by TFE, resulting in tetramer dissociation. To gain insight into this possibility, we performed a study on the effect of TFE on the KcsA tetramer in a lipid environment. First, the effect of TFE on the structure of KcsA in lipid micelles was studied, using SDSPAGE, cicular dichroism, and protease treatment. Subsequently, the effect of the lipid environment on the KcsA tetramer dissociation was determined, varying from lipid micelles to bilayers containing nonbilayer lipids. To understand how lipids can mediate the effect of TFE, the interaction of TFE with pure lipid systems was studied by carboxyfluorescein-leakage and ${ }^{31} \mathrm{P}$ NMR and ${ }^{2} \mathrm{H}$ NMR measurements.

Our results indicate that lipids stabilize the KcsA tetramer against TFE-induced tetramer dissociation, in a way that depends on the effective overall shape of the lipids. The stabilizing effect increases going from a detergent molecule with an inverted conical shape, to a more cylindrical PC or PG and finally to the conically shaped nonbilayer lipid PE. We suggest that PE increases the membrane lateral pressure in the acyl chain region due to its conical shape, and that it thereby stabilizes the KcsA tetramer. TFE can lower this lateral pressure and in that way contribute to tetramer dissociation.

\section{MATERIALS AND METHODS}

Materials. $\mathrm{Ni}^{2+}$-NTA agarose was obtained from Qiagen. Bio-Beads SM-2 Adsorbent was from Bio-Rad Laborotories. $n$-Dodecyl- $\beta$-D-maltoside (DDM) was purchased from Anatrace Inc. 1,2-Dielaidoyl-sn-glycero-3-phosphoethanolamine (DEPE), 1,2-dioleoyl-sn-glycero-3-phosphocholine (DOPC), and 1,2-dioleoyl-sn-glycero-3-phophoglycerol (DOPG) were purchased from Avanti Polar Lipids Inc. 1,2Dioleoyl-sn-glycero-3-phosphoethanolamine (DOPE) was obtained from Lipoid GMBH (Ludwigshafen, Germany). TFE was obtained from Merck. Deuterium depleted water was from Cambridge Isotope laboratories (Cambridge, MA). DOPC labeled with two deuterium atoms on both acyl chains at the $\mathrm{C}_{11}$ position $\left({ }^{2} \mathrm{H}_{4}\right.$-DOPC) was synthesized as described previously $(28,29)$.

KcsA Purification and Reconstitution. KcsA with an $\mathrm{N}$-terminal His-tag was expressed and purified as described previously (19) and stored at $4{ }^{\circ} \mathrm{C}$ in a buffer containing 20 mM Hepes, pH 7.5, $100 \mathrm{mM} \mathrm{NaCl}, 5 \mathrm{mM} \mathrm{KCl}, 300 \mathrm{mM}$ imidazole and $1 \mathrm{mM}$ DDM, typically in a concentration of $\pm 1 \mathrm{mg} / \mathrm{mL}$. C-terminally truncated $\operatorname{KcsA}(\Delta 125-160)$ was obtained by chymotrypsin treatment $(250 \mu \mathrm{g} / \mathrm{mL}, 60 \mathrm{~min}$, room temperature) of wild-type KcsA (30).
Large unilamellar vesicles (LUVs) were prepared by extrusion (Hope 1985): dry lipid films were hydrated in 50 $\mathrm{mM}$ Tris-HCl, pH 7.5, $200 \mathrm{mM} \mathrm{NaCl}, 15 \mathrm{mM} \mathrm{KCl}$, frozen and thawed 5 times, and extruded 5 times using 200-nm membrane filters (Anatop 10, Whatman, UK). KcsA was reconstituted in different lipid mixtures as described previously (19), with a protein concentration of $\pm 0.1 \mathrm{mg} / \mathrm{mL}$ and a protein-lipid ratio of 1:1000.

TFE-Induced Dissociation of the KcsA Tetramer. Fifteenmicroliter samples of KcsA, in DDM or reconstituted in vesicles, in a typical concentration of $\pm 0.1 \mathrm{mg} / \mathrm{mL}$ protein, were incubated with a variable concentration of TFE for $1 \mathrm{~h}$ at room temperature. Subsequently, an acetone precipitation was performed to remove TFE and phospholipids. For this, $1 \mathrm{~mL}$ of cold acetone $\left(-20^{\circ} \mathrm{C}\right)$ was added to each sample and the samples were incubated at $-20^{\circ} \mathrm{C}$ for at least $2 \mathrm{~h}$. After centrifugation (5 min, eppendorf centrifuge, 14000 $\mathrm{rpm}$ ) the pellets were dried by incubation for $15 \mathrm{~min}$ at 37 ${ }^{\circ} \mathrm{C}$ and subsequently resuspended in SDS-PAGE sample buffer. After SDS-PAGE and Coomassie brilliant blue staining, the gels were scanned by a densitometer (Bio-Rad Laboratories) and quantified with the program Quantity One.

TFE-induced dissociation of the KcsA tetramer in Escherichia coli membranes was studied with vesicles that were prepared by sonication after lysozyme treatment of KcsA overexpressing $E$. coli cells, as is normally done for the purification of KcsA $(19,26)$.

Circular Dichroism. Purified KcsA was dialyzed overnight against $1 \mathrm{mM}$ DDM, $50 \mathrm{mM}$ Tris- $\mathrm{HCl}, \mathrm{pH} 7.5,15$ $\mathrm{mM} \mathrm{KCl}$ at $4{ }^{\circ} \mathrm{C}$ to remove interfering substances such as imidazole. Before a measurement, KcsA was incubated during $1 \mathrm{~h}$ with a variable concentration of TFE at room temperature. Measurements were carried out on a Jasco J-810 spectropolarimeter, using a 1-mm path length cell, $0.2-\mathrm{nm}$ interval, $0.5 \mathrm{~s}$ response time and a scan speed of 10 $\mathrm{nm} / \mathrm{min}$. As a blank, the CD spectrum of the dialysis buffer (with TFE) was measured. Spectra were corrected for the volume increase due to the presence of TFE. The CD spectra in millidegrees (mdeg) were converted to mean residue ellipticity $\left([\theta], \operatorname{deg} \mathrm{cm}^{2} \mathrm{dmol}^{-1}\right.$ residue $\left.^{-1}\right)$ by $[\theta]=$ (mdeg*0.1*MRW) $/\left(P^{*} C\right)$, in which MRW is the mean residue weight (protein mean weight/number of residues), $P$ is the path length $(\mathrm{cm})$, and $C$ is the protein concentration $(\mathrm{mg} / \mathrm{mL})$. The CD spectra were analyzed with the analysis programs CONTINLL $(31,32)$ and $\operatorname{CDSSTR}(33,34)$ on the website DICHROWEB (35), using the reference protein sets 4 and 7, with the assumption that the protein concentration was not changed during dialysis (prior to the TFE incubation to remove imidazole) and incubation with TFE.

Carboxyfluorescein (CF) Leakage Experiments. Large unilamellar vesicles (10 $\mathrm{mM}$ phospholipids) were prepared as described above in buffer containing $50 \mathrm{mM} \mathrm{CF}, 25 \mathrm{mM}$ Tris-HCl, pH 7.5, $100 \mathrm{mM} \mathrm{NaCl}, 7.5 \mathrm{mM} \mathrm{KCl}$. Subsequently, the $\mathrm{CF}$ outside the vesicles was removed by a Sephadex G-25 spin column, prepared in $50 \mathrm{mM}$ Tris- $\mathrm{HCl}$, pH 7.5, $200 \mathrm{mM} \mathrm{NaCl}, 15 \mathrm{mM} \mathrm{KCl}$ (buffer A).

For the leakage experiments, a continuously stirred $10 \times$ $4 \mathrm{~mm}$ cuvette was filled with $1 \mathrm{~mL}$ of buffer A and placed in an SLM-Aminco SPF-500 C fluorometer. The excitation wavelength was set at $491 \mathrm{~nm}$ and the emission wavelength was at $517 \mathrm{~nm}$, both with a bandwidth of $2.5 \mathrm{~nm}$. A variable amount of TFE was added to $20 \mu \mathrm{L}$ of the CF vesicles in an 

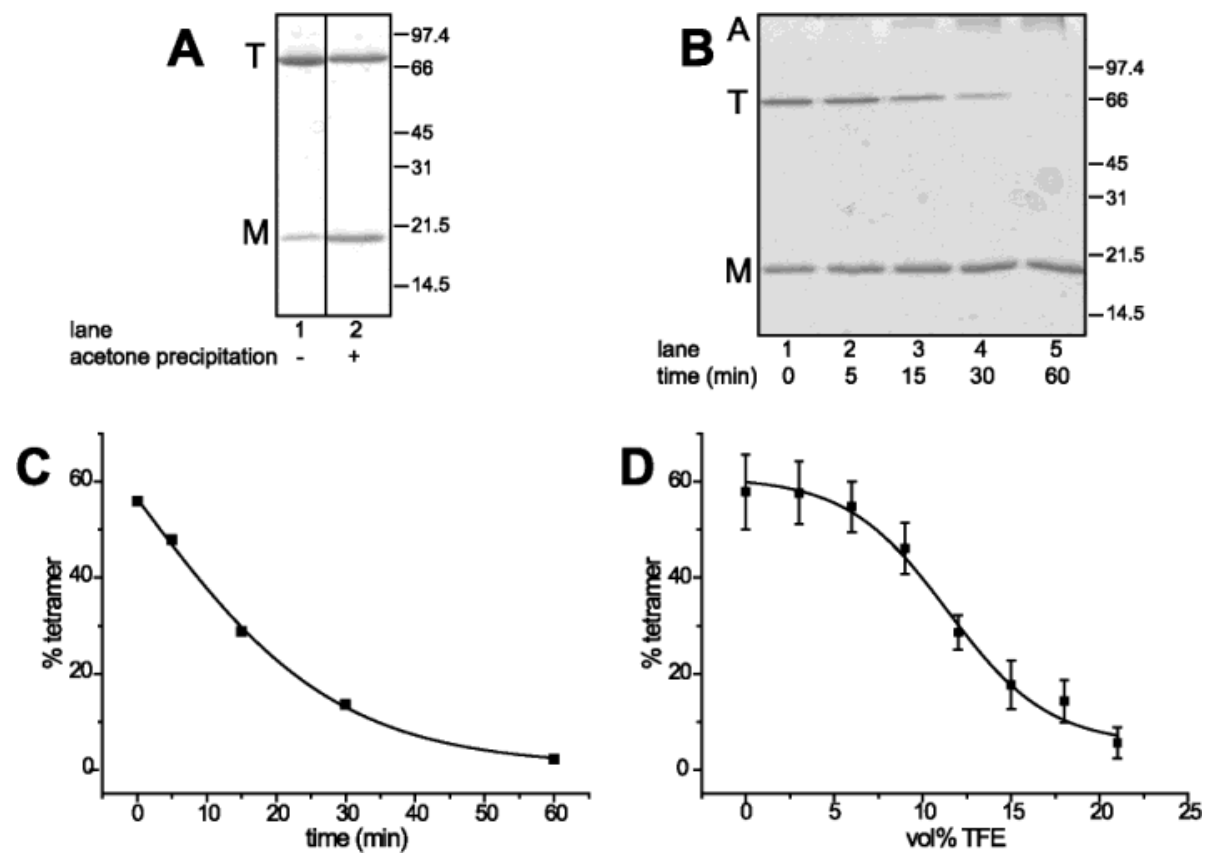

FIGURE 1: TFE-induced dissociation of the KcsA tetramer in $1 \mathrm{mM}$ DDM as shown on SDS-PAGE. (A) SDS-PAGE of KcsA before and after acetone precipitation (no TFE added). Monomeric (M) and tetrameric (T) KcsA are indicated and a protein size marker (in kDa) is shown on the right. (B) SDS-PAGE of KcsA, incubated with 20 vol \% TFE for 0, 5, 15, 30, or 60 min (with subsequent acetone precipitation prior to SDS-PAGE). Monomeric (M) and tetrameric (T) and aggregated (A) KcsA are indicated and a protein size marker (in kDa) is shown on the right. (C) Quantification of the SDS-PAGE gel shown in panel B, as determined by densitometry. The \% of tetramer, relative to the total amount of KcsA in each lane, was calculated. (D) Effect of the TFE concentration of the KcsA tetramer dissociation in $1 \mathrm{mM}$ DDM. KcsA in $1 \mathrm{mM}$ DDM was incubated with various concentrations of TFE for $1 \mathrm{~h}$ at room temperature. Samples were analyzed by SDS-PAGE after acetone precipitation and visualized by Coomassie staining. The \% of tetramer, relative to the total amount of KcsA in each lane, was calculated based on densitometry. The standard deviation is based on at least three different experiments. The data points were fit with a sigmoidal curve fit.

eppendorf tube. After vortexing, 25 vol \% of this sample (5 $\mu \mathrm{L}$ for a sample without TFE) was added to the cuvette, and the fluorescence was measured immediately. The percentage of $\mathrm{CF}$ leakage was calculated after subtracting the fluorescence without TFE, relative to a sample in which full leakage was induced by the addition of $1 \%$ Triton $\mathrm{X}-100$ $(\mathrm{w} / \mathrm{v})$.

NMR Measurements. NMR samples were prepared by solvent evaporation of phospholipid solutions in chloroform. The residual solvent was removed under high vacuum for at least $12 \mathrm{~h}$. Subsequently, the lipids were hydrated with buffer (50 mM Tris-HCl, pH 7.5, $200 \mathrm{mM} \mathrm{NaCl,} 15 \mathrm{mM}$ $\mathrm{KCl}$ ), containing a variable amount of TFE. For ${ }^{2} \mathrm{H}$ NMR samples, the buffer was prepared with deuterium-depleted water. The samples for ${ }^{31} \mathrm{P}$ NMR consisted of $30 \mu \mathrm{mol}$ of lipid, in a total volume of $1 \mathrm{~mL}$. The samples for ${ }^{2} \mathrm{H}$ NMR consisted of $2 \mu \mathrm{mol}$ of lipid, in a total volume of $100 \mu \mathrm{L}$. The samples were stored at $4{ }^{\circ} \mathrm{C}$ before measuring. NMR spectra were recorded on an Avance $500 \mathrm{WB}$ spectrometer (Bruker, Germany). ${ }^{31} \mathrm{P}$ NMR spectra were recorded at 202.48 MHz using a high resolution 10-mm broad band probe with broad band gated proton decoupling. The recycling delay was $1.5 \mathrm{~s}$ and the $\pi / 4$ pulse width was $7 \mu \mathrm{s}$. Typically, 2000 scans were recorded. An exponential multiplication with a line-broadening factor of $100 \mathrm{~Hz}$ was used before performing the Fourier transformation. Temperature scans were recorded in steps of $2{ }^{\circ} \mathrm{C}$. Samples were allowed to equilibrate for $10 \mathrm{~min}$ after each temperature increase. At the end of the measurements, samples were cooled to their initial temperature and reanalyzed to check the reversibility of the temperature effects on the sample. ${ }^{2} \mathrm{H}$ NMR spectra were recorded at $76.8 \mathrm{MHz}$, using a high power 8-mm probe and a quadrupolar echo sequence (36) with a $6 \mu \mathrm{s} 90^{\circ}$ pulse and a recycling delay of $100 \mathrm{~ms}$. Typically, 30000 scans were acquired for samples containing $2 \mu \mathrm{mol}$ of ${ }^{2} \mathrm{H}_{4}$-DOPC. A line broadening of up to $100 \mathrm{~Hz}$ was used.

\section{RESULTS}

TFE-induced Tetramer Dissociation of KcsA in Detergent. The potassium channel KcsA forms an extremely stable tetramer, even in SDS (26). Therefore, it is possible to analyze the dissociation of the tetramer by SDS-PAGE. Figure 1A, lane 1, shows a typical SDS-PAGE gel for purified KcsA, with a tetramer-band running at a molecular mass of $67 \mathrm{kDa}$ and a minor monomer band around $18 \mathrm{kDa}$. Since direct SDS-PAGE analysis was not possible for samples containing TFE (no protein bands were visible after staining, especially for samples containing more than $30 \mathrm{vol}$ $\%$ TFE), we decided to perform an acetone precipitation after the incubation. This resulted in a slightly increased amount of monomer (compare lane 1 and 2 of Figure 1A for KcsA without and with acetone precipitation). First, the effect of TFE on KcsA in detergent micelles was studied. Figure 1B shows a typical result for KcsA in $1 \mathrm{mM}$ DDM, incubated with 20 vol \% TFE for different time periods. The quantification of this SDS-PAGE gel is shown in Figure 1C. These data show that TFE induces a time-dependent dissociation of the KcsA tetramer. Although this mainly results in monomeric KcsA, some protein aggregrates also are formed (see for example Figure 1B, top of the gel in lanes 4 and 5). The tetramer dissociation seems to be a slow process with a half-life of $\pm 15 \mathrm{~min}$. For all other experiments with KcsA 


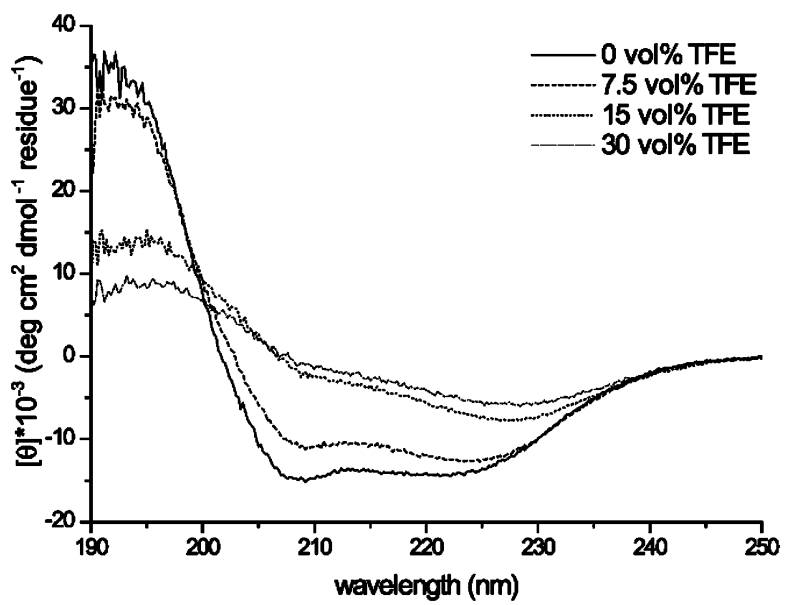

FIGURE 2: Circular dichroism spectra of KcsA in $1 \mathrm{mM}$ DDM after incubation with various concentrations of TFE. CD spectra were recorded after $1 \mathrm{~h}$ incubation of KcsA with TFE at room temperature. Prior to this incubation, the protein was dialyzed against $1 \mathrm{mM}$ DDM, $50 \mathrm{mM}$ Tris- $\mathrm{HCl}, \mathrm{pH} 7.5,15 \mathrm{mM} \mathrm{KCl}$ at 4 ${ }^{\circ} \mathrm{C}$ to remove interfering substances such as imidazole. Spectra were corrected for the blank and for the volume increase due to TFE.

Table 1: Secondary Structure Content of KcsA in $1 \mathrm{mM}$ DDM, Incubated with Different Concentrations of $\mathrm{TFE}^{a}$

\begin{tabular}{cccccc}
\hline vol $\%$ & & & & & \\
TFE & $\alpha$-helix & $\beta$-strands & turns & unordered & NRMSD \\
\hline 0 & $51 \pm 3$ & $11 \pm 1$ & $15 \pm 1$ & $23 \pm 3$ & $0.047 \pm 0.031$ \\
7.5 & $46 \pm 1$ & $15 \pm 2$ & $15 \pm 1$ & $24 \pm 2$ & $0.038 \pm 0.025$ \\
15 & $21 \pm 7$ & $26 \pm 4$ & $23 \pm 4$ & $30 \pm 3$ & $0.088 \pm 0.071$ \\
\hline
\end{tabular}

${ }^{a}$ Structure analysis was performed for the averaged CD spectra, corrected for the blanc and for volume increase due to TFE. The shown data are the average and standard deviation of the results of two different analysis methods, CONTINLL and CDSSTR, each performed with two different reference protein sets, using DICHROWEB (35). The NRMSD is the normalized root-mean-square deviation.

an incubation time of $60 \mathrm{~min}$ was used. Figure 1D shows that for this incubation time, $12 \mathrm{vol} \%$ TFE is necessary to dissociate $50 \%$ of the initial amount of KcsA tetramers, while around $20 \mathrm{vol} \%$ TFE is sufficient for complete dissociation of the protein oligomer. Removal of TFE by dialysis (in the presence of lipid vesicles) did not result in tetramer formation (data not shown), indicating that this dissociation process is irreversible. This is in apparent contrast to previous observations that TFE-dissociated KcsA can be refolded albeit with a low efficiency (24). However, these latter studies were done under different conditions and in the presence of SDS after removal of TFE (24). Therefore, this seems to be an independent refolding process that is not directly related to the TFE-induced tetramer dissocation.

Next, the effect of TFE on the secondary and tertiary structure of KcsA was investigated. To obtain information about the secondary structure, circular dichroism spectroscopy was used. The resulting spectra are shown in Figure 2, with the estimated secondary structure content given in Table 1. Without TFE, the protein contained a high content of $\alpha$-helical structure, in good agreement with earlier published $\mathrm{CD}$ spectra of KcsA (26) and with the structure of the protein $(22,23)$. Incubation with $7.5 \mathrm{vol} \%$ TFE resulted only in a small change in the $\mathrm{CD}$ spectrum, corresponding to a small decrease in the $\alpha$-helical content and an increase in the content of $\beta$-strands. However, when the TFE concentration

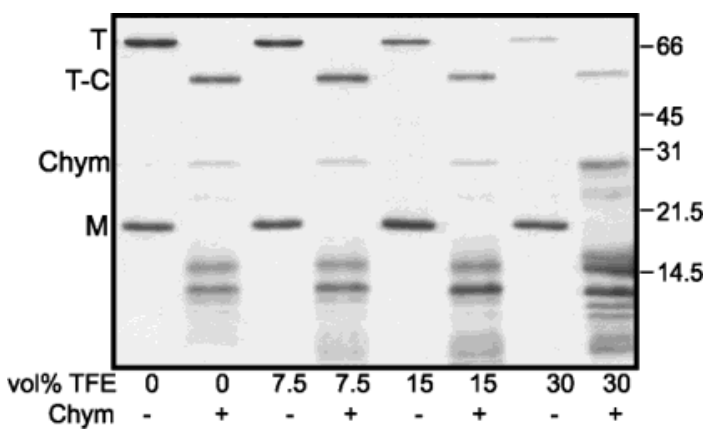

FIGURE 3: Protease resistance of KcsA in the presence of various concentrations of TFE. KcsA in buffer containing $1 \mathrm{mM}$ DDM was incubated with various concentrations of TFE and $0.25 \mathrm{mg} / \mathrm{mL}$ chymotrypsin for $1 \mathrm{~h}$ at room temperature. Samples were analyzed by SDS-PAGE (after acetone precipitation) and visualized by Coomassie staining. Monomeric (M), tetrameric (T), chymotrypsin cleaved tetrameric $(\mathrm{T}-\mathrm{C}) \mathrm{KcsA}$, and chymotrypsin (Chym) are indicated and a protein size marker (in $\mathrm{kDa}$ ) is shown on the right.

was increased to $15 \mathrm{vol} \%$, a large change in the CD spectrum was observed, indicating a dramatic loss of $\alpha$-helical structure, while the content of $\beta$-strands and unordered structure was increased. Further increasing the TFE concentration to $30 \mathrm{vol} \%$ did not result in any large changes in the CD spectrum. Although we were not able to obtain good analysis results for this spectrum, we suggest that the secondary structure content was similar as for 15 vol \% TFE. These data show that in KcsA TFE induces a large change in secondary structure, from $\alpha$-helices to $\beta$-strands and unordered structures. The change in secondary structure (between 7.5 and $15 \mathrm{vol} \%$ TFE) seems to occur simultaneously with the tetramer-to-monomer transition of KcsA (around 12 vol \% TFE).

To obtain additional information on the structure of KcsA during the TFE-induced unfolding, a protease treatment was used. When a protein is correctly folded, a protease can only cleave at exposed cleavage sites. However, when a protein loses its tertiary structure, more cleavage sites will be exposed, resulting in more different protein fragments visible on SDS-PAGE. We used the protease chymotrypsin, which is also active in the presence of TFE (at least till $30 \mathrm{vol} \%$ ). The results are shown in Figure 3. Without TFE, chymotrypsin only cleaves off the C-terminal domain of the tetrameric KcsA (30), resulting in a shifted tetramer-band $(\mathrm{T}-\mathrm{C})$ as is shown in Figure 3, lane 2. The absence of the monomer-band and the appearance of two fragments of \pm 12 and $15 \mathrm{kDa}$ indicates that these fragments result from chymotrypsin cleavage of the monomer. The relatively large size of these fragments suggests that monomeric KcsA still contains contains some tertiary structure, since only a limited number of cleavage sites is exposed. When the TFE concentration is increased, this results in a decrease in the amount of tetramer, as was also shown in Figure 1D. For all conditions, only one shifted tetramer band is visible after chymotrypsin treatment, of which the intensity is similar to that of the tetramer band of the samples without chymotrypsin, indicating that there is no loss of tertiary structure before the tetramer dissociation. In contrast, the fragment pattern of monomeric KcsA changes when the TFE concentration is increased, with more different bands of increasing intensity, especially when going to $30 \mathrm{vol} \%$ TFE. This indicates that after the tetramer dissociation, the monomer 


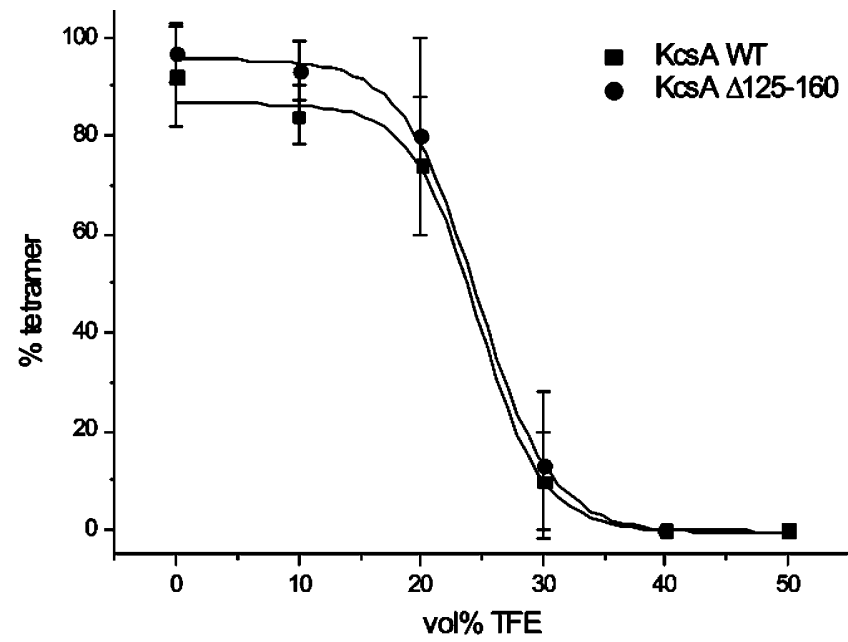

FIgURE 4: Effect of the C-terminal domain of KcsA on the TFEinduced tetramer dissociation. WT KcsA and C-terminally truncated KcsA $(\Delta 125-160$, obtained by chymotrypsin treatment of WT KcsA) were reconstituted in DOPC and incubated with various concentrations of TFE during $1 \mathrm{~h}$ at room temperature. Samples were analyzed by SDS-PAGE after acetone precipitation. Coomassie-stained gels were quantified by densitometry. The $\%$ of tetramer, relative to the total amount of protein in each lane, was calculated, based on at least three different experiments. The data points were fit with a sigmoidal curve fit.

still contains some tertiary structure, which is mainly lost at 30 vol \% TFE.

TFE-Induced Dissociation of the KcsA Tetramer in a Lipid Bilayer. Subsequently, we studied the effect of the lipid environment on the TFE-induced dissociation of the KcsA tetramer. As a first indication, it was determined which part of KcsA is important for the tetramer stability: the transmembrane domain, which is in contact with lipids, or the large C-terminal cytoplasmic domain. Therefore, wild-type KcsA was compared with KcsA from which the C-terminal domain was removed by chymotrypsin treatment. For this variant, it was shown before that it has a reduced thermostability $(19,30)$. However, removal of the C-terminal domain had no effect on the stability of KcsA during TFEinduced tetramer dissociation (tested after reconstitution in DOPC, as shown in Figure 4), suggesting that TFE mainly acts on the transmembrane domain of KcsA.

Next, we investigated the role of protein-lipid interactions in the TFE-induced tetramer dissociation of KcsA, by comparing the KcsA tetramer dissociation in different lipid environments. As shown in Figure 5, reconstitution of KcsA in a bilayer of PC resulted in a large increase in the tetramer stability: the dissociating TFE concentration is increased from 12 vol \% for KcsA in DDM to 25 vol \% for KcsA in a PC bilayer. No significant difference between PC and PC/ PG was observed, indicating no special role of $\mathrm{PG}$ in the TFE-induced tetramer dissociation. In contrast, the nonbilayer lipid PE has a large effect on the KcsA tetramer dissociation: more than 40-45 vol \% TFE is needed to dissociate half of the KcsA tetramers in PE/PG (7:3), while this is 25 vol \% TFE for PC/PG (7:3) and pure PC.

To determine how KcsA is stabilized in a biological membrane instead of a pure lipid bilayer, membrane vesicles of KcsA overexpressing $E$. coli cells were incubated with different concentrations of TFE. Because of the overexpression, the KcsA bands are well visible on a normal Coo-

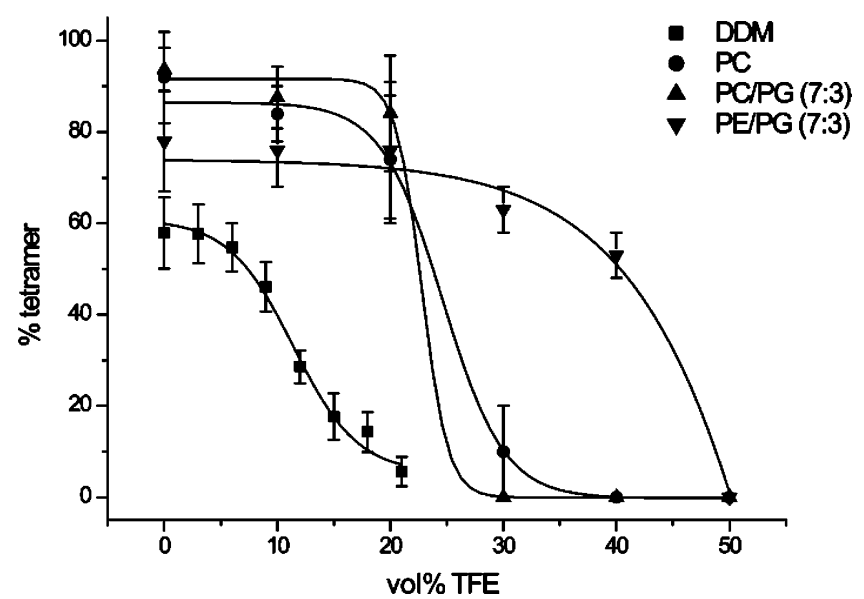

FIGURE 5: Effect of lipids on the TFE-induced dissociation of the KcsA tetramer. KcsA in $1 \mathrm{mM}$ DDM, or reconstituted in DOPC, DOPC/DOPG (7:3), or DOPE/DOPG (7:3) was incubated with various concentrations of TFE during $1 \mathrm{~h}$ at room temperature. Samples were analyzed by SDS-PAGE after acetone precipitation. Coomassie-stained gels were quantified by densitometry. The $\%$ of tetramer, relative to the total amount of protein in each lane, was calculated, based on at least three different experiments (results for DDM are the same as in Figure 1D). The data points were fit with a sigmoidal curve fit.

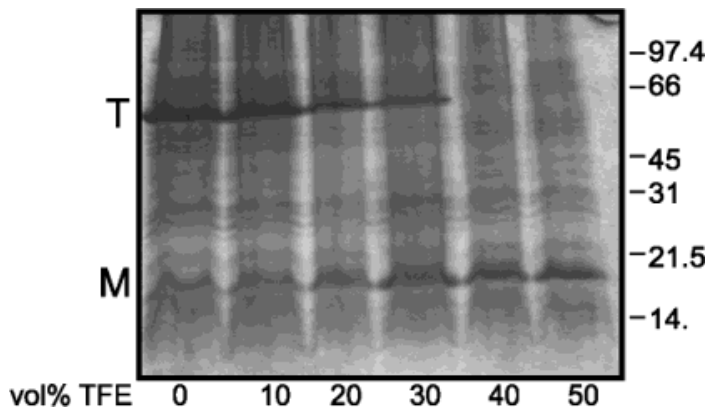

FIGURE 6: TFE-induced dissociation of the KcsA tetramer in $E$. coli membranes. KcsA was overexpressed in E. coli and membrane vesicles were isolated as described in Materials and Methods. Membrane vesicles were incubated with TFE during $1 \mathrm{~h}$ at room temperature. Samples were analyzed by SDS-PAGE (after acetone precipitation) and visualized by Coomassie staining. Monomeric (M) and tetrameric (T) KcsA are indicated and a protein size marker (in $\mathrm{kDa}$ ) is shown on the right.

massie-stained SDS-PAGE gel, as is shown in Figure 6. Without TFE, KcsA is mainly tetrameric. With increasing concentrations of TFE, the tetramer band disappears and the monomer band is becoming more intense. The concentration of TFE that is needed for KcsA tetramer dissociation $( \pm 30$ vol \%) is comparable to what was observed for KcsA reconstituted in pure lipid bilayers.

Interaction of TFE with the Lipid Bilayer. Because of the observed lipid dependency of the KcsA tetramer dissociation, we next studied the interaction of TFE with pure lipid systems, using different biophysical techniques.

First, the effect of TFE on the bilayer integrity was studied. Therefore, carboxyfluorescein $(\mathrm{CF})$ leakage experiments were performed for lipid bilayers of PC, PC/PG (7:3), and PE/ PG (7:3). Figure 7 shows that a PC bilayer becomes leaky around 10 vol \% TFE. For a PC/PG (7:3) bilayer, only a slightly higher amount of TFE is needed, but a PE/PG (7:3) bilayer is clearly much more stable in the presence of TFE: around $20 \mathrm{vol} \%$ TFE is needed to cause $50 \%$ leakage. These results show that the lipid dependency of leakage is similar 


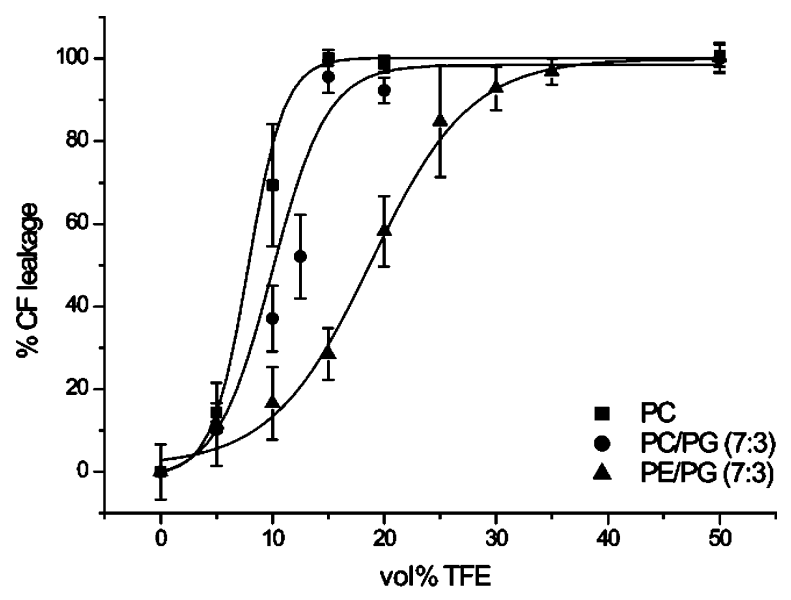

FIGURE 7: Carboxyfluorescein leakage of membrane vesicles induced by TFE. TFE was added to carboxyfluorescein-loaded vesicles (10 $\mathrm{mM}$ phospholipids, without KcsA). The leakage was determined immediately after dilution with buffer in the cuvette (see Materials and Methods). The standard deviation is based on at least three different measurements. The data points were fit with a sigmoidal curve fit.

to the lipid dependency of the KcsA tetramer dissociation (Figure 5), although the TFE concentration needed to obtain CF leakage is much lower than the concentration that causes KcsA tetramer dissociation.

Subsequently, ${ }^{31} \mathrm{P}$ NMR measurements were performed for phospholipids in buffer with different concentrations of TFE. ${ }^{31} \mathrm{P} \mathrm{NMR}$ is a convenient method to determine the aggregate structure formed by the phospholipids. The results for $\mathrm{PC} /$ PG (7:3) and PE/PG (7:3) are shown in Figure 8. For PC/ PG without TFE the ${ }^{31} \mathrm{P}$-spectrum is typical of phospholipids in the liquid crystalline $\left(\mathrm{L}_{\alpha}\right)$ bilayer phase (37). The two peaks in the spectrum arise from the two different phospholipids $\mathrm{PC}$ and $\mathrm{PG}$, in which $\mathrm{PG}$ has the smallest chemical shift anisotropy (CSA). Also at 10 and 20 vol \% TFE such bilayer spectrum was observed, although now the peaks of the two different phospholipids could not be distinguished anymore because of a loss of resolution. Furthermore, the CSA decreased from $41 \mathrm{ppm}$ for 0 vol \% TFE (as measured for the PC component) to $27 \mathrm{ppm}$ for $20 \mathrm{vol} \% \mathrm{TFE}$, indicating a larger motional freedom of the phospholipid headgroups. At TFE concentrations of $30 \mathrm{vol} \%$ and higher the spectrum was fully isotropic. In these spectra, the peaks for the two phospholipids are separated again (low field peak PG, high field peak PC). Such an isotropic signal demonstrates high motional freedom of the phospholipid molecules in all directions, but can originate from many different structures. Since the sample had the appearance of a clear nonviscous solution, we suggest that at these TFE concentrations the membrane is completely dissolved. For DOPC similar results were obtained, although in this case an isotropic spectrum was measured only at $40 \mathrm{vol} \%$ TFE and higher (data not shown). The results for DOPE/DOPG were totally different, as shown in Figure 8B: for these samples an isotropic signal was observed only at concentrations of 60 vol \% TFE and higher, but even at 70 vol \% TFE only a small isotropic signal was observed, while the $\mathrm{L}_{\alpha}$ bilayer spectrum was still dominating. Hence, even at 70 vol \% TFE most of the PE/PG (7:3) bilayer is still intact and only a small fraction of the phospholipids is dissolved. These results support the conclusion from the CF-leakage experiments that
PE increases the stability of the lipid bilayer compared to PC, while PG has almost no effect.

Next, we determined the effect of TFE on the $\mathrm{L}_{\alpha}$ bilayerto-hexagonal $\left(\mathrm{H}_{\mathrm{II}}\right)$ phase transition of $\operatorname{DEPE}(38,39)$, to get an indication about the localization of TFE in the lipid bilayer. If TFE would primarily be located between the acyl chains, the hexagonal phase would be promoted by the resulting increase in chain volume, while the opposite result can be expected when TFE is located in the lipid bilayer interface region. A ${ }^{31} \mathrm{P}$ NMR temperature scan was used to determine the effect of $10 \mathrm{vol} \%$ TFE on this phase behavior of DEPE, as is shown in Figure 9. Figure 9A shows the result for DEPE without TFE, which undergoes a $\mathrm{L}_{\alpha}-\mathrm{to}_{\mathrm{H}} \mathrm{H}_{\mathrm{II}}$ phase transition at $54{ }^{\circ} \mathrm{C}$, in good agreement with literature (39). This phase transition was reversible (data not shown). In the presence of 10 vol \% TFE, a transition to an isotropic spectrum is observed between 40 and $48{ }^{\circ} \mathrm{C}$. This spectrum probably originates from a cubic phase, since the signal stayed isotropic after cooling to the initial temperature (data not shown) and the sample was a transparent viscous solution. Lipids that form a cubic phase have a more cylindrical effective shape due to a larger headgroup compared to lipids in the hexagonal phase (40). Since occupancy of space in the interface is equivalent to increasing the headgroup size of the lipids, our results suggests that TFE localizes mainly in the headgroup region of the lipid bilayer, resulting in the observed preference for the cubic instead of the $\mathrm{H}_{\text {II }}$ phase.

Next, we investigated how this interaction affects the lipid acyl chains. Therefore, the effect of TFE on the acyl chain order was measured by ${ }^{2} \mathrm{H}$ NMR with DOPC, deuterated at the 11-position of the oleoyl-chains. The quadrupolar splitting $\left(\Delta v_{\mathrm{q}}\right)$, measured as the distance between the two peaks in the ${ }^{2} \mathrm{H}$ NMR spectrum, is a measure of the chain order, with increasing chain order resulting in larger values of $\Delta v_{\mathrm{q}}$. Figure 10 shows that already 10 vol $\%$ TFE has a dramatic effect on the quadrupolar splitting, which is decreased from 6 to $2 \mathrm{kHz}$. With $20 \mathrm{vol} \%$ TFE the spectrum is isotropic, indicating a low order and a high flexibility of the acyl chains within the bilayer. These results show also that the structure of the acyl chain region is strongly affected by the presence of TFE.

\section{DISCUSSION}

In this paper, we investigated which interactions stabilize the oligomeric structure of the potassium channel KcsA in a lipid environment against TFE-induced dissociation. In the introduction, we hypothesized that besides protein-protein interactions, protein-lipid interactions especially are important for the tetramer stabilization, and we suggested that these protein-lipid interactions can be disturbed by the presence of TFE. Here we discuss the data that we collected to investigate this hypothesis.

Effect of TFE on the Structure of KcsA. The observation that TFE causes dissociation of the KcsA tetramer was surprising, because of the extremely high stability of this tetramer, as reported previously (26). This high stability arises not only from interactions between hydrophobic parts of the subunits, but also from hydrogen bonding between polar residues in the selectivity filter $(12,17,22)$. The finding that removal of the $\mathrm{C}$-terminal domain had no effect on the 


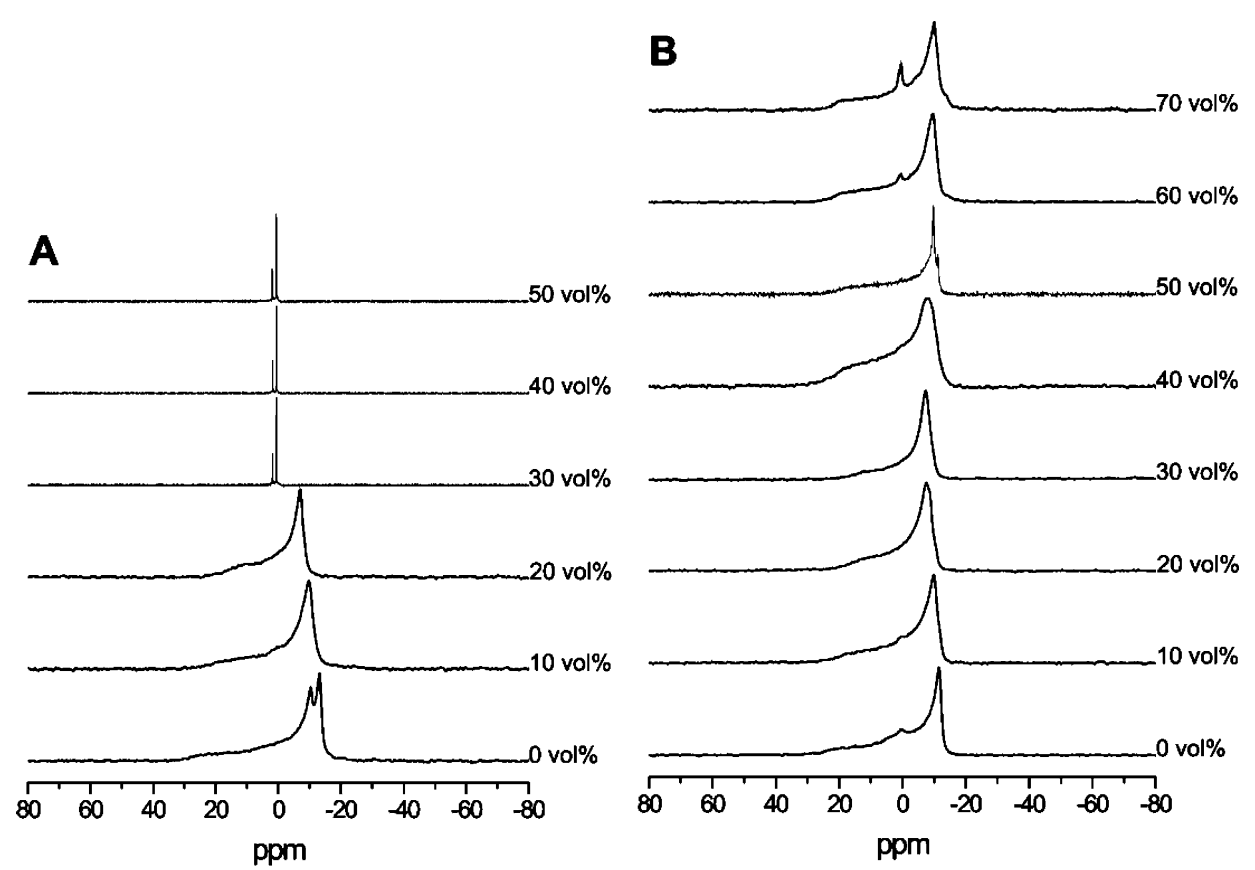

FIGURE 8: ${ }^{31} \mathrm{P}$ NMR spectra of DOPC/DOPG (7:3, panel A) and DOPE/DOPG (7:3, panel B) dispersions in the presence of an increasing amount of TFE. Dry lipid films were hydrated with buffer containing TFE. Separate samples were prepared for each concentration of TFE. ${ }^{31} \mathrm{P}$ NMR spectra were recorded at room temperature. The volume percentage of TFE is given for each spectrum.
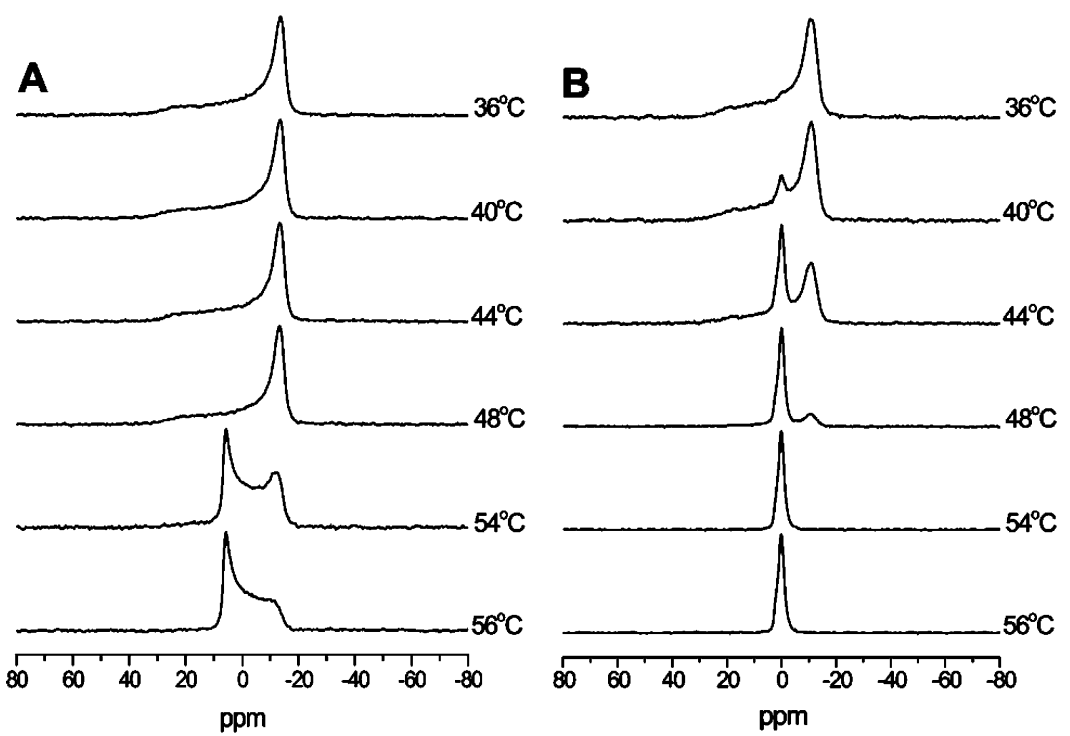

FIGURE 9: The effect of TFE on the phase transition of DEPE as measured by ${ }^{31} \mathrm{P}$ NMR. ${ }^{31} \mathrm{P}$ NMR spectra are shown of DEPE in the absence (A) or presence (B) of $10 \mathrm{vol} \%$ TFE at different temperatures. Most important spectra are shown in this figure.

tetramer stability suggests that these stabilizing interactions occur mainly in the transmembrane domain of the protein. Apparently, TFE weakens both these hydrophobic and polar interactions.

What happens to the KcsA protein structure during this dissociation process? During the tetramer dissociation, the secondary and tertiary structure of KcsA also is largely changed, as indicated by the CD (Figure 2 and Table 1) and protease treatment data (Figure 3 ). These changes seem to occur mainly simultaneous with the tetramer dissociation, suggesting that the tetramer dissociation occurs in a twostage process, and not via a loosely packed tetramer as intermediate. During the tetramer dissociation, the secondary structure of KcsA is partly changed from $\alpha$-helix to $\beta$-sheet and unordered structure (Table 1). This was striking, since it is generally assumed that TFE stabilizes helical conforma- tions (27). However, this may not be the case for hydrophobic proteins (41). We suggest that when TFE induces KcsA tetramer dissociation, the hydrophobic surface of the transmembrane helices is exposed. TFE clusters may then cooperatively associate with this surface to prevent contact with water (27). In that way, the protein can stay in the monomeric form. However, the exposed hydrophobic protein parts can also cluster to form protein aggregates, as seen in Figure $1 \mathrm{~B}$. The $\beta$-sheet formation is probably caused by this aggregate formation, similar to what was found previously for several peptides (27). The protease treatment data (Figure 3 ) indicate that directly after the tetramer dissociation the monomeric KcsA still contains some tertiary structure. However, when the TFE concentration is further increased, TFE also weakens these tertiary interactions. 


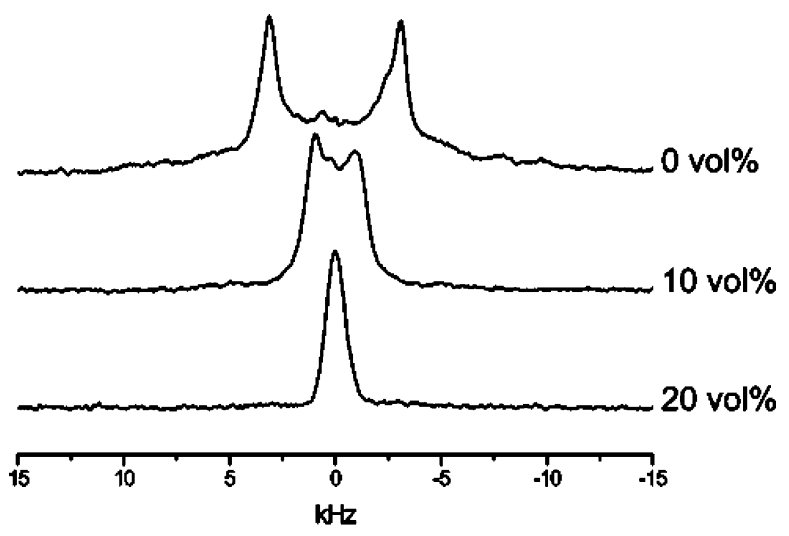

FIGURE 10: ${ }^{2} \mathrm{H}$ NMR spectra of ${ }^{2} \mathrm{H}_{4}$-DOPC in the presence of 0-20 vol \% TFE. Dry lipid films of $2 \mu \mathrm{mol}$ of ${ }^{2} \mathrm{H}_{4}$-DOPC were hydrated with $100 \mu \mathrm{L}$ of buffer containing TFE. Separate samples were prepared for each concentration of TFE. ${ }^{2} \mathrm{H}$ NMR spectra were recorded at room temperature.

Thus, it is possible that TFE exerts its effect directly on KcsA. However, another possibility for the interaction of TFE with KcsA is an indirect interaction via the lipid or detergent molecules surrounding the protein. To understand the possible role of this indirect interaction between TFE and KcsA via the surrounding lipids, it is necessary to understand first how TFE interacts with the lipids themselves.

Effect of TFE on the Lipid Bilayer. We observed that TFE changes the phase transition of DEPE from an $\mathrm{L}_{\alpha}$ bilayerhexagonal to an $\mathrm{L}_{\alpha}$ bilayer-isotropic (probably cubic) phase transition. The effective shape of molecules in a cubic phase is more cylindrical compared to lipid molecules in the hexagonal phase (40). Since space filling between the lipid headgroups is equivalent to increasing the lipid headgroup size, we concluded that TFE localizes mainly in the headgroup region of the lipid bilayer. This localization is similar to that of ethanol in the lipid bilayer $(42,43)$. The insertion of TFE in the membrane interface results in a lower lipid packing of the membrane. This is not only in the headgroup region, but also in the acyl chain region, as indicated by the lower quadrupolar splitting measured by ${ }^{2} \mathrm{H}$ NMR (Figure 10). As a result of this lower packing density, the membrane gets leaky (Figure 7). At high concentrations of TFE, the bilayer seems to dissolve completely, as indicated by the isotropic signal in ${ }^{31} \mathrm{P}$ NMR (Figure 8).

The extent of the effect of TFE on the lipid structure depends on the lipid composition, as indicated by the CFleakage (Figure 7) and ${ }^{31} \mathrm{P}$ NMR (Figure 8) experiments. While there was no significant stabilizing effect of the anionic lipid PG compared to PC, we observed a large stabilizing effect of the nonbilayer lipid PE. The bilayer lipids PC and PG both have a large headgroup, resulting in an overall cylindrical shape, while the small headgroup of the nonbilayer lipid PE results in an overall conical shape. Because of this shape, the presence of nonbilayer lipids in a lipid bilayer results in a lower packing density in the headgroup region and a higher packing density in the acyl chain region. We have shown previously that the lower packing density in the headgroup region results in the presence of interfacial insertion sites, e.g., for membrane insertion of the catalytic domain of leader peptidase (44). We suggest also that TFE can insert into these interfacial insertion sites. Since the size of these insertion sites is larger for PE compared to PC (44), a bilayer containing PE can accommodate a higher amount of TFE inserting in the headgroup region. This implies that a bilayer structure can be maintained up to higher concentrations of TFE, which we observed.

Effect of TFE on KcsA in a Lipid Environment. When we studied the effect of the surrounding lipids on the KcsA tetramer stability, we found that the tetramer was strongly stabilized by a lipid bilayer compared to detergent micelles. Introducing the nonbilayer lipid PE in the lipid bilayer further stabilized the tetramer relative to a bilayer containing PC, while there was no effect of the anionic lipid PG, similar to what we found for the effect of TFE on pure lipid bilayers. For the pure lipid systems, we observed a correlation between the bilayer stability and the effective lipid shape, with an increasing stability going from cylindrical bilayer lipids to more conical nonbilayer lipids. For KcsA, we observe a similar correlation, which can even be extended to the inverted conical detergent molecules. In the presence of these molecules, which have a shape opossite to that of the conical lipid PE, the KcsA stability is reduced as compared to that in cylindrical bilayer lipids.

The lack of effect of the anionic lipid PG is remarkable, since previously often an interaction with PG has been observed $(19,24,25)$. PG was found to be important for efficient membrane assembly and thermostability of the KcsA tetramer (19). The interaction with PG even stayed intact in $50 \%$ TFE (25). PG is an anionic phospholipid that probably interacts with KcsA via electrostatic interactions. Since TFE can promote ionic interactions (45), it leaves the interaction with the anionic PG intact (25), while weakening the hydrophobic interactions between the KcsA subunits and between KcsA and PE. Although the importance of the interaction with PG is obvious, this interaction seems not to play a role in the stability of the KcsA tetramer against TFEinduced dissocation. The finding that the stabilization of the KcsA tetramer was similar for KcsA reconstituted in a lipid bilayer and for KcsA in E. coli membranes further suggests that also in vivo the tetramer stabilization occurs mainly via the surrounding lipids and that no protein factors are involved.

On the basis of these data, we cannot exclude a direct interaction between TFE and the KcsA protein. It might be that the presence of the lipid bilayer limits the exposure of the transmembrane helices of KcsA to TFE, and therefore stabilizes the KcsA tetramer. However, the finding that both the bilayer structure and the KcsA tetramer stability depend on the lipid shape suggests an indirect effect of TFE on KcsA via the surrounding lipids. This is supported by our previous observation that also during heat-induced KcsA tetramer dissociation the tetramer is stabilized by a surrounding lipid bilayer compared to (mixed) micelles (19). Since the TFEinduced tetramer dissociation seems to be a two-stage process, the question arises about what exactly triggers this dissociation. Table 2 shows an overview of the effects of TFE on KcsA and the lipid bilayer. This shows that the TFE concentration at which membrane leakage is induced is much lower than the concentration needed for tetramer dissociation. On the other hand, TFE dissolves the membrane at much higher concentrations. This suggests that neither membrane leakage nor dissolving the bilayer triggers the tetramer to dissociate. The tetramer dissociation most likely is related to an effect on the membrane lipid packing, for which we 
Table 2: Summary of the Effects of TFE on KcsA and the Lipid Bilayer $^{a}$

\begin{tabular}{cccc}
\hline & $\begin{array}{c}\text { DOPC } \\
(\text { vol \%) }\end{array}$ & $\begin{array}{c}\text { DOPC/DOPG } \\
(7: 3)(\text { vol \% })\end{array}$ & $\begin{array}{c}\text { DOPE/DOPG } \\
(7: 3)(\text { vol \% })\end{array}$ \\
\hline $50 \%$ KcsA tetramer & 25 & 25 & 40 \\
$\begin{array}{c}\text { dissociated } \\
50 \% \text { CF-leakage }\end{array}$ & 8 & 10 & 20 \\
$\begin{array}{c}\text { isotropic signal } \\
\text { 31P NMR }\end{array}$ & 40 & 30 & $\geq 60$ \\
\hline
\end{tabular}

${ }^{a}$ The vol $\%$ of TFE at which $50 \%$ of the KcsA tetramer is dissociated (as shown in Figure 5), 50\% of the carboxyfluorescein is leaked out (Figure 7), a transition was observed from a bilayer spectrum to an isotropic signal in ${ }^{31} \mathrm{P}$ NMR (Figure 8).

observed large effects of TFE (as indicated by ${ }^{2} \mathrm{H}$ NMR, Figure 10).

Membrane Lateral Pressure. A unifying principle to explain indirect membrane protein-lipid interactions is the membrane lateral pressure. The membrane lateral pressure varies with the depth within the bilayer $(46,47)$. A large negative lateral pressure is localized at the interface between the headgroups and acyl chain region, due to the interfacial tension. This is compensated by a positive lateral pressure in the headgroup region due to repulsions between the headgroups and a positive lateral pressure in the acyl chain region due to acyl chain repulsions $(46,47)$. The resulting lateral pressure profile is sensitive to changes in the lipid composition (48). Such changes in the lateral pressure have been suggested to modulate protein function (46), e.g., for bacteriorhodopsin (49) and the ion channel forming peptide alamethicin $(50,51)$. For this peptide, it was also calculated that changes in the lateral pressure profile affect the size distribution of the peptide oligomers, in qualitative agreement with experimental results (51). Therefore, we suggest that also for the tetrameric KcsA, the tetramer stability depends on the lateral pressure in the membrane. We suggest that especially a high lateral pressure in the acyl chain region has a stabilizing effect, since this can press the tetramer subunits together. The tetramer dissociation will only be affected by the membrane lateral pressure if this is accompanied by a change in depth-dependent change in the cross-sectional area $(46,52)$. We suggest that this indeed occurs during tetramer dissociation. This might occur by flipping out the pore region, similar as was shown for the monomer during topological membrane insertion $(21)$ and by flipping out the amphipatic N-terminal domain, which might go out of the membrane interface because of the presence of TFE in the headgroup region of the membrane. As a result the overall shape of the KcsA monomer would be more cylindrical compared to the subunits of the KcsA tetramer. It has been proposed that nonbilayer lipids, because of their conical shape, increase the lateral pressure in the acyl chain region, while decreasing the lateral pressure in the headgroup region, compared to a membrane containing bilayer lipids $(53,54)$. This is in agreement with our finding that nonbilayer lipids have a strong stabilizing effect. Moreover, it has been calculated that low molecular weight alcohols, similar to TFE, also give rise to a changed lateral pressure profile, with an increased lateral pressure in the upper part of the acyl chain region $(55,56)$, which further supports our suggestion. Since TFE inserts mainly in the headgroup region of the membrane, and gives rise to a lower

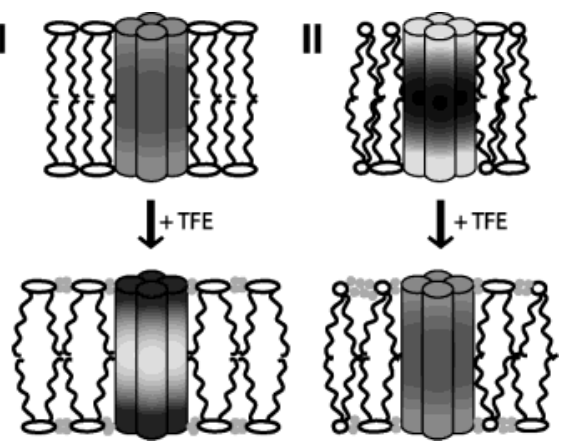

FIGURE 11: Summarizing model of the effect of the surrounding lipids and TFE on the lateral pressure acting on KcsA. For simplicity, a KcsA monomer is represented as a cylinder. The coloring of the cylinders indicates the positive lateral pressure acting on the KcsA tetramer, with dark coloring representing a high positive lateral pressure, and light coloring indicating a low positive lateral pressure. For simplicity, the negative lateral pressure at the interface between the headgroups and acyl chains is not indicated in the model. The KcsA tetramer is shown in a bilayer containing bilayer lipids (I) or nonbilayer lipids (II). The effect of addition of TFE ( $\pm 20 \mathrm{vol} \%)$ on the lateral pressure is also shown for both systems. On the basis of Figure 4, we suggest that at a concentration of $\pm 20 \mathrm{vol} \%$ TFE, the acyl chain lateral pressure in a bilayer of PE/PG (7:3) is similar to the acyl chain lateral pressure in a membrane of PC/PG (7:3) without TFE, since in both systems an additional 25 vol \% TFE is needed to induce KcsA tetramer dissociation.

acyl chain ordering, TFE most likely increases the lateral pressure in the headgroup region, while lowering the pressure between the acyl chains.

A practical implication of our results is that the assay described here might be useful for studying the effect of different detergents and lipid additions from the viewpoint of membrane protein crystallization. One might be able to derive general conclusions as to which detergent/lipid composition provides the highest lateral pressure, and may therefore be most suited for membrane protein crystallization, for example, for bicelle forming detergent/lipid mixtures (57).

Summarizing Model. To summarize our conclusions and suggestions, a model is shown in Figure 11. This figure describes in a semiquantitative manner the membrane lateral pressure acting on the KcsA tetramer, as a function of the lipid environment and the presence of TFE. In this figure, we only show the relative height of the lateral pressure acting in the lipid headgroup region and the acyl chain region. Going from a bilayer containing only bilayer lipids (I) and a bilayer containing nonbilayer lipids (II), the lateral pressure in the acyl chain region increases, resulting in an increased stability of the oligomeric protein. This is compensated by a decrease in the lateral pressure in the lipid headgroup region. TFE inserts between the lipid headgroups. For both types of lipids, this results in a lower lateral pressure in the acyl chain region, compensated by an increased lateral pressure in the headgroup. As a result, the stability of the oligomeric protein complex is decreased by the presence of TFE.

\section{REFERENCES}

1. Russ, W. P., and Engelman, D. M. (2000) The GxxxG motif: a framework for transmembrane helix-helix association. J. Mol. Biol. 296, 911-919.

2. Choma, C., Gratkowski, H., Lear, J. D., and DeGrado, W. F. (2000) Asparagine-mediated self-association of a model transmembrane helix. Nat. Struct. Biol. 7, 161-166. 
3. Zhou, F. X., Merianos, H. J., Brunger, A. T., and Engelman, D. M. (2001) Polar residues drive association of polyleucine transmembrane helices. Proc. Natl. Acad. Sci. U.S.A. 98, 2250-2255.

4. Nussberger, S., Dorr, K., Wang, D. N., and Kuhlbrandt, W. (1993) Lipid-protein interactions in crystals of plant light-harvesting complex. J. Mol. Biol. 234, 347-356.

5. Tsukihara, T., Aoyama, H., Yamashita, E., Tomizaki, T., Yamaguchi, H., Shinzawa Itoh, K., Nakashima, R., Yaono, R., and Yoshikawa, S. (1996) The whole structure of the 13-subunit oxidized cytochrome $c$ oxidase at $2.8 \AA$. Science 272,1136-1144.

6. Luecke, H., Schobert, B., Richter, H. T., Cartailler, J. P., and Lanyi, J. K. (1999) Structure of bacteriorhodopsin at $1.55 \AA$ resolution. J. Mol. Biol. 291, 899-911.

7. McAuley, K. E., Fyfe, P. K., Ridge, J. P., Isaacs, N. W., Cogdell, R. J., and Jones, M. R. (1999) Structural details of an interaction between cardiolipin and an integral membrane protein. Proc. Natl. Acad. Sci. U.S.A. 96, 14706-14711.

8. Orr, J. W., and Newton, A. C. (1992) Interaction of protein kinase $\mathrm{C}$ with phosphatidylserine. 2. Specificity and regulation. Biochemistry 31, 4667-4673.

9. Killian, J. A. (1998) Hydrophobic mismatch between proteins and lipids in membranes. Biochim. Biophys. Acta 1376, 401-415.

10. van der Does, C., Swaving, J., van Klompenburg, W., and Driessen, A. J. (2000) Nonbilayer lipids stimulate the activity of the reconstituted bacterial protein translocase. J. Biol. Chem. 275 , 2472-2478.

11. Rietveld, A. G., Koorengevel, M. C., and de Kruijff, B. (1995) Nonbilayer lipids are required for efficient protein transport across the plasma membrane of Escherichia coli. EMBO J. 14, 55065513.

12. Irizarry, S. N., Kutluay, E., Drews, G., Hart, S. J., and Heginbotham, L. (2002) Opening the KcsA K+ channel: tryptophan scanning and complementation analysis lead to mutants with altered gating. Biochemistry 41, 13653-13662.

13. Sansom, M. S., Shrivastava, I. H., Bright, J. N., Tate, J., Capener, C. E., and Biggin, P. C. (2002) Potassium channels: structures, models, simulations. Biochim. Biophys. Acta 1565, 294-307.

14. Kelly, B. L., and Gross, A. (2003) Potassium channel gating observed with site-directed mass tagging. Nat. Struct. Biol. 10, 280-284.

15. Nimigean, C. M., Chappie, J. S., and Miller, C. (2003) Electrostatic tuning of ion conductance in potassium channels. Biochemistry 42, 9263-9268.

16. Shen, N. V., Chen, X., Boyer, M. M., and Pfaffinger, P. J. (1993) Deletion analysis of $\mathrm{K}+$ channel assembly. Neuron. 11, 67-76.

17. Splitt, H., Meuser, D., Borovok, I., Betzler, M., and Schrempf, H. (2000) Pore mutations affecting tetrameric assembly and functioning of the potassium channel KcsA from Streptomyces lividans. FEBS Lett. 472, 83-87.

18. van Dalen, A., Schrempf, H., Killian, J. A., and de Kruijff, B. (2000) Efficient membrane assembly of the KcsA potassium channel in Escherichia coli requires the protonmotive force. EMBO Rep. 1, 340-346.

19. van Dalen, A., Hegger, S., Killian, J. A., and de Kruijff, B. (2002) Influence of lipids on membrane assembly and stability of the potassium channel KcsA. FEBS Lett. 525, 33-38.

20. van Dalen, A., van der Laan, M., Driessen, A. J., Killian, J. A., and de Kruijff, B. (2002) Components required for membrane assembly of newly synthesized $\mathrm{K}+$ channel KcsA. FEBS Lett. $511,51-58$.

21. Umigai, N., Sato, Y., Mizutani, A., Utsumi, T., Sakaguchi, M., and Uozumi, N. (2003) Topogenesis of two transmembrane type $\mathrm{K}+$ channels, Kir 2.1 and KcsA. J. Biol. Chem. 278, 4037340384.

22. Doyle, D. A., Morais Cabral, J., Pfuetzner, R. A., Kuo, A., Gulbis, J. M., Cohen, S. L., Chait, B. T., and MacKinnon, R. (1998) The structure of the potassium channel: molecular basis of $\mathrm{K}+$ conduction and selectivity. Science 280, 69-77.

23. Cortes, D. M., Cuello, L. G., and Perozo, E. (2001) Molecular architecture of full-length KcsA: role of cytoplasmic domains in ion permeation and activation gating. J. Gen. Physiol. 117, 165180.

24. Valiyaveetil, F. I., Zhou, Y., and MacKinnon, R. (2002) Lipids in the structure, folding, and function of the KcsA $\mathrm{K}+$ channel. Biochemistry 41, 10771-10777.

25. Demmers, J. A., van Dalen, A., de Kruijff, B., Heck, A. J., and Killian, J. A. (2003) Interaction of the K+ channel KcsA with membrane phospholipids as studied by ESI mass spectrometry. FEBS Lett. 541, 28-32.
26. Cortes, D. M., and Perozo, E. (1997) Structural dynamics of the Streptomyces lividans $\mathrm{K}+$ channel (SKC1): oligomeric stoichiometry and stability. Biochemistry 36, 10343-10352.

27. Buck, M. (1998) Trifluoroethanol and colleagues: cosolvents come of age. Recent studies with peptides and proteins. Q. Rev. Biophys. 31, 297-355.

28. Chupin, V., Killian, J. A., and de Kruijff, B. (1987) 2H-nuclear magnetic resonance investigations on phospholipid acyl chain order and dynamics in the gramicidin-induced hexagonal HII phase. Biophys. J. 51, 395-405.

29. Farren, S. B., Sommerman, E. F., and Cullis, P. R. (1984) Production of specifically $2 \mathrm{H}$-labeled unsaturated phospholipids in gram quantities: a convenient synthesis of [11,11-2H2] oleic acid. Chem. Phys. Lipids 34, 279-286.

30. Perozo, E., Cortes, D. M., and Cuello, L. G. (1999) Structural rearrangements underlying $\mathrm{K}+$-channel activation gating. Science $285,73-78$.

31. Provencher, S. W., and Glockner, J. (1981) Estimation of globular protein secondary structure from circular dichroism. Biochemistry $20,33-37$

32. van Stokkum, I. H., Spoelder, H. J., Bloemendal, M., van Grondelle, R., and Groen, F. C. (1990) Estimation of protein secondary structure and error analysis from circular dichroism spectra. Anal. Biochem. 191, 110-118.

33. Manavalan, P., and Johnson, W. C., Jr. (1987) Variable selection method improves the prediction of protein secondary structure from circular dichroism spectra. Anal. Biochem. 167, 76-85.

34. Sreerama, N., and Woody, R. W. (2000) Estimation of protein secondary structure from circular dichroism spectra: comparison of CONTIN, SELCON, and CDSSTR methods with an expanded reference set. Anal. Biochem. 287, 252-260.

35. Lobley, A., Whitmore, L., and Wallace, B. A. (2002) DICHROWEB: an interactive website for the analysis of protein secondary structure from circular dichroism spectra. Bioinformatics 18, 211212.

36. Davis, J. H. (1983) The description of membrane lipid conformation, order and dynamics by $2 \mathrm{H}-\mathrm{NMR}$. Biochim. Biophys. Acta 737, 117-171.

37. Cullis, P. R., and de Kruijff, B. (1979) Lipid polymorphism and the functional roles of lipids in biological membranes. Biochim. Biophys. Acta 559, 399-420.

38. Veiro, J. A., Khalifah, R. G., and Rowe, E. S. (1989) The polymorphic phase behavior of dielaidoylphosphatidylethanolamine. Effect of $n$-alkanols. Biochim. Biophys. Acta 979, 251256.

39. Kooijman, E. E., Chupin, V., de Kruijff, B., and Burger, K. N. (2003) Modulation of membrane curvature by phosphatidic acid and lysophosphatidic acid. Traffic 4, 162-174.

40. Cherezov, V., Clogston, J., Misquitta, Y., Abdel-Gawad, W., and Caffrey, M. (2002) Membrane protein crystallization in meso: lipid type-tailoring of the cubic phase. Biophys. J. 83, 33933407.

41. Lehrman, S. R., Tuls, J. L., and Lund, M. (1990) Peptide $\alpha$-helicity in aqueous trifluoroethanol. Correlations with predicted $\alpha$-helicity and the secondary structure of the corresponding regions of bovine growth hormone. Biochemistry 29, 5590-5596.

42. Barry, J. A., and Gawrisch, K. (1994) Direct NMR evidence for ethanol binding to the lipid-water interface of phospholipid bilayers. Biochemistry 33, 8082-8088.

43. Holte, L. L., and Gawrisch, K. (1997) Determining ethanol distribution in phospholipid multilayers with MAS-NOESY spectra. Biochemistry 36, 4669-4674.

44. van den Brink-van der Laan, E., Dalbey, R. E., Demel, R. A. Killian, J. A., and de Kruijff, B. (2001) Effect of nonbilayer lipids on membrane binding and insertion of the catalytic domain of leader peptidase. Biochemistry 40, 9677-9684.

45. MacPhee, C. E., Perugini, M. A., Sawyer, W. H., and Howlett, G. J. (1997) Trifluoroethanol induces the self-association of specific amphipathic peptides. FEBS Lett. 416, 265-268.

46. Cantor, R. S. (1997) Lateral pressures in cell membranes: a mechanism for modulation of protein function. J. Phys. Chem. B $101,1723-1725$

47. Marsh, D. (1996) Components of the lateral pressure in lipid bilayers deduced from HII phase dimensions. Biochim. Biophys. Acta 1279, 119-123.

48. Cantor, R. S. (1999) Lipid composition and the lateral pressure profile in bilayers. Biophys. J. 76, 2625-2639.

49. Curran, A. R., Templer, R. H., and Booth, P. J. (1999) Modulation of folding and assembly of the membrane protein bacteriorhodop- 
sin by intermolecular forces within the lipid bilayer. Biochemistry $38,9328-9336$.

50. Keller, S. L., Bezrukov, S. M., Gruner, S. M., Tate, M. W., Vodyanoy, I., and Parsegian, V. A. (1993) Probability of alamethicin conductance states varies with nonlamellar tendency of bilayer phospholipids. Biophys. J. 65, 23-27.

51. Cantor, R. S. (2002) Size distribution of barrel-stave aggregates of membrane peptides: influence of the bilayer lateral pressure profile. Biophys. J. 82, 2520-255.

52. Bezrukov, S. M. (2000) Functional consequences of lipid packing stress. Curr. Opin. Colloid Interface Sci. 5, 237-243.

53. de Kruijff, B. (1997) Lipid polymorphism and biomembrane function. Curr. Opin. Chem. Biol. 1, 564-569.
54. de Kruijff, B. (1997) Lipids beyond the bilayer. Nature 386, 129130.

55. Cantor, R. S. (1997) The lateral pressure profile in membranes: a physical mechanism of general anesthesia. Biochemistry 36 , $2339-2344$.

56. Cantor, R. S. (1998) The lateral pressure profile in membranes: a physical mechanism of general anesthesia. Toxicol Lett. 100, $451-458$.

57. Faham, S., and Bowie, J. U. (2002) Bicelle crystallization: a new method for crystallizing membrane proteins yields a monomeric bacteriorhodopsin structure1. J. Mol. Biol. 316, 1-6.

BI036129D 